\begin{tabular}{|c|l|}
\hline Title & $\begin{array}{l}\text { Electron acceleration in gas by impul se electric field and its application to selective promotion of an electron-molecule } \\
\text { reaction }\end{array}$ \\
\hline Author(s) & Sugawara, Hirotake; Sakai, Y osuke \\
\hline Citation & $\begin{array}{l}\text { Journal of Physics D: A pplied Physics, 36(16), 1994.2000 } \\
\text { https://doi.org/10.1088/0022-3727/36/6/311 } \\
\text { Journal of Physics D: A pplied Physics, 36(16), 2003, p.1994.2000 }\end{array}$ \\
\hline Issue Date & 2003-08-21 \\
\hline Doc URL & http://hdl.handle.net/2115/766 \\
\hline Rights & Copyright (c) 2003 IOP Publishing Ltd. \\
\hline Type & article (author version) \\
\hline File Information & sugawarajpd-36.pdf \\
\hline
\end{tabular}

Instructions for use 


\title{
Electron acceleration in gas by impulse electric field and its application to selective promotion of an electron-molecule reaction
}

\author{
Hirotake Sugawara and Yosuke Sakai \\ Division of Electronics and Information Engineering, Graduate School of \\ Engineering, Hokkaido University, Sapporo 060-8628 Japan
}

\begin{abstract}
We have simulated electron acceleration under short intense impulse electric fields of $0.1-1 \mathrm{kV} \mathrm{cm}{ }^{-1}$ strength and $\sim 1 \mathrm{~ns}$ duration in $\mathrm{N}_{2}$ at $65.5 \mathrm{~Pa}$. The electron acceleration is completed in a practically collisionless condition when the impulse duration is comparable with or shorter than the electron mean free time. On the basis of this feature, we have proposed a way to produce a group of monoenergetic electrons. The electron energy after acceleration is controllable by the impulse strength and duration, and we have demonstrated selective promotion of the excitation producing $\mathrm{N}_{2}\left(\mathrm{~A}^{3} \Sigma_{\mathrm{u}}^{+}\right)$metastable excited molecules. The selectivity has been quantified as the efficiency of the electron energy utilized for the target reaction, and efficiencies of $20-30 \%$ have been achieved in this simulation. These values are higher than those of electron swarms in DC equilibrium, in which the efficiency is at most $10 \%$. The electron behaviour under impulse electric fields and the dependence of the energy efficiency on the impulse profile have been investigated.
\end{abstract}

PACS numbers: 52.80.-s Electric discharges

Published source: Journal of Physics D: Applied Physics, 36(16), pp. 1994-2000 (2003) 


\section{Introduction}

In applications of weakly ionised plasmas, high voltage pulses are expected as an advantageous means of dynamic plasma control utilizing non-equilibrium electron behaviour. Penetrante et al (1997) mentioned the use of fast-rising voltage pulses $(<10 \mathrm{~ns}$ risetime) as an effective way to increase the mean electron energy for high yields of electron-molecule reactions in decomposition of air pollutants. Macheret et al (2002) evaluated the input power required to sustain air plasmas by repetitive voltage pulses (0.1-5 ns duration), and reported its lower budgets compared with the DC mode. In investigations of dielectric barrier discharges for ultraviolet light sources, as well, the advantage of the use of short voltage pulses has been referred to as a key to obtain high radiation efficiencies (e.g., Vollkommer and Hitzschke (1997, 1998), Carman and Mildren (2003)).

Sugawara et al (1999) pointed out a theoretical possibility that electron acceleration under well-controlled impulse electric fields leads to selective promotion of a specific electron-molecule reaction. Electrons in gas can be accelerated by an impulse electric field in a practically collisionless condition when the impulse duration is comparable with or shorter than the electron mean free time. The electron energy after acceleration is controllable by the impulse field strength and duration, and monoenergetic electrons are obtained when the electron temperature before acceleration is low enough. Let us call this technique the impulse-field electron acceleration (IFEA) method. Impulse fields required for the IFEA method are, e.g., in the order of $\sim 1 \mathrm{kV} \mathrm{cm}^{-1}$ strength and $\sim 1$ ns duration at gas pressures of around 1 Torr. Recent progress in pulsedpower apparatus is achieving a satisfactory capability for this requirement. Impulse voltages of $10-100 \mathrm{kV}$ amplitude and $\sim 10 \mathrm{~ns}$ duration are available nowadays. Anikin et al (1998) observed ionisation waves in $\mathrm{N}_{2}$ induced by impulse voltages of $15.5 \mathrm{kV}$ amplitude and $25 \mathrm{~ns}$ duration in a pressure range of $0.1-16$ Torr. Related to this work, Pancheshnyi et al (1999) measured the population of excited $\mathrm{N}_{2}$ particles and Starikovskaia and Starikovskii (2001) modelled the electron energy distribution function. Katsuki et al (2001) reported on streamer discharges in atmospheric-pressure air induced by $10 \mathrm{~ns}$ impulses with voltages up to $140 \mathrm{kV}$. Although there may currently be technical difficulties in precise control of the impulse profiles, investigation of the electron behaviour under a short impulse electric field would be informative for applications of impulse-driven plasmas.

In this paper, we simulate electron behaviour during the IFEA and subsequent electron-molecule collisions. As the target reaction to be promoted, we take the excitation to produce $\mathrm{N}_{2}\left(\mathrm{~A}^{3} \Sigma_{\mathbf{u}}^{+}\right)$metastable excited molecules. The efficiency of the electron energy utilized for the target reaction is compared with those of electron swarms in drift equilibrium under DC electric fields and monoenergetic electron beams. We investigate the dependence of the efficiency on the impulse profile. 


\section{Theory}

In the beginning, we consider collision-free electron acceleration. After that, electronmolecule collisions during the acceleration is taken into account.

Letting $\boldsymbol{v}=\left(v_{x}, v_{y}, v_{z}\right)$ be the electron velocity and $E(t)$ be the electric field applied along the $-x$-direction during a period $0 \leq t \leq T$, the velocity increase $\Delta v_{x}=v_{x}(T)-v_{x}(0)$ of an electron given by $E(t)$ is written as

$$
\Delta v_{x}=\int_{0}^{T} \frac{e}{m} E(t) \mathrm{d} t \equiv \frac{e}{m} E_{\max } T_{\text {eff }}
$$

Here, $e$ and $m$ are the electronic charge and mass. $E_{\max }$ and $T_{\text {eff }}$ are parameters introduced to characterize the strength and duration of $E(t) ; E_{\max }$ is the maximum value of $E(t)$, and $T_{\text {eff }}$ is the effective impulse duration defined as $T_{\text {eff }}=\int_{0}^{T} E(t) \mathrm{d} t / E_{\max } \cdot \Delta v_{x}$ is common for all of the electrons under $E(t)$. Thus the electron velocity distribution function (EVDF) $f\left(v_{x}, v_{y}, v_{z}, t\right)$ shifts parallelly in the $+v_{x}$-direction in velocity space by the acceleration:

$$
f\left(v_{x}, v_{y}, v_{z}, T\right)=f\left(v_{x}-\Delta v_{x}, v_{y}, v_{z}, 0\right) .
$$

When the electron temperature before acceleration is low, the electrons are concentrated around $\boldsymbol{v}=0$ and the electrons after acceleration are also concentrated around $\left(\Delta v_{x}, 0,0\right)$; monoenergetic electrons are obtained through this collective shift. With an appropriate choice of $E_{\max }$ and $T_{\text {eff }}$, one can feed electrons into a specific velocity (energy) range in which target collisions occur efficiently. Especially in the case in which the target collision cross-section, $q_{\text {target }}$, lays alone in the range, the target process can be promoted selectively. Figure 1 illustrates an aspect of the IFEA aiming at $q_{\text {target }}$ in such an energy range that is free from another cross-section $q^{\prime}$ which impairs the selectivity. Here, $f\left(v_{x}, t\right)$ in figure 1 is the projection of $f\left(v_{x}, v_{y}, v_{z}, t\right)$ to the $v_{x}$ axis of velocity space:

$$
f\left(v_{x}, t\right)=\int_{-\infty}^{\infty} \int_{-\infty}^{\infty} f\left(v_{x}, v_{y}, v_{z}, t\right) \mathrm{d} v_{y} \mathrm{~d} v_{z} .
$$

In the presence of gas molecules, some of the electrons drop out of the collective acceleration because of scattering. The ratio of the dropout electrons, i.e. the collision probability $P_{\text {coll }}$ during $0 \leq t \leq T$, is given as

$$
P_{\text {coll }}=1-\exp \left(-\int_{0}^{T} N q_{\text {total }}(v(t)) v(t) \mathrm{d} t\right),
$$

where $q_{\text {total }}$ is the total collision cross-section as a function of $v=|\boldsymbol{v}|$ and $N$ is the number density of gas molecules. This evaluation can be simplified as follows when the electron mean free time $\tau$ or the electron mean free path $\lambda$ is known:

$$
P_{\text {coll }} \approx 1-\exp (-T / \tau) \text { or } \quad P_{\text {coll }} \approx 1-\exp (-L / \lambda),
$$

where $L$ is the flight distance of an electron during the acceleration. $P_{\text {coll }}$ is satisfactorily small when IFEA is completed within $T<\tau$ or $L<\lambda$.

Here, a small $N$ is also preferable for reducing $P_{\text {coll }}$. However, decrease in the collision frequency of the target reaction after acceleration and increase in the electron 


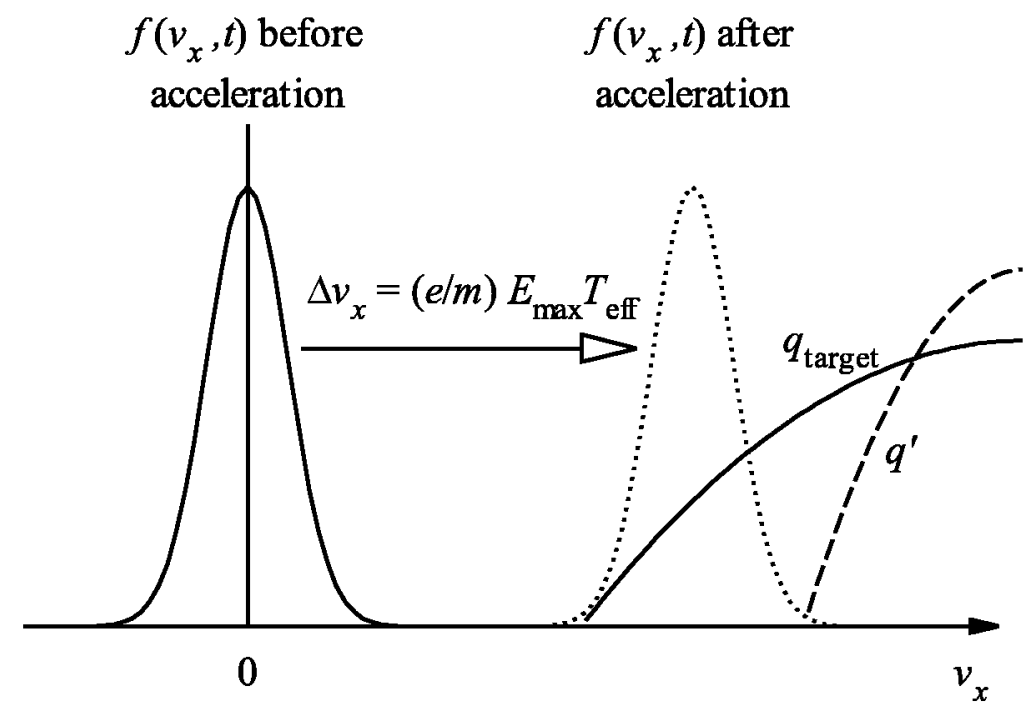

Figure 1. Schematic of electron acceleration by impulse electric field. $f\left(v_{x}, t\right)$, the distribution function of $v_{x} ; q_{\text {target }}$, the electron collision cross-section of the target process; and $q^{\prime}$, another cross-section to be avoided so as not to impair the selectivity.

diffusivity, both of which lower the volume reaction rate of the target process, should be taken into account when one makes a practical choice of $N$.

In order to achieve the electron acceleration over the onset energy $\epsilon_{\text {target }}$ of the target reaction, the impulse field must be intense inversely proportional to the flight time or distance. For an electron with velocity $\boldsymbol{v}=\left(\Delta v_{x}, 0,0\right)$, which is the representative (central) electron velocity after acceleration, the requirement on the kinetic energy $\epsilon=\frac{1}{2} m|\boldsymbol{v}|^{2}$ is given as follows from equation (1):

$$
\epsilon=\frac{e^{2}}{2 m}\left(E_{\max } T_{\text {eff }}\right)^{2}>\epsilon_{\text {target }} .
$$

\section{Simulation conditions}

\subsection{Target reaction and energy efficiency}

We simulate the IFEA in $\mathrm{N}_{2}$. Excitation to produce $\mathrm{N}_{2}\left(\mathrm{~A}^{3} \Sigma_{\mathbf{u}}^{+}\right)$metastable excited molecules is chosen as the target process. This species is known for its long lifetime of over $10 \mathrm{~s}$ (Radzig and Smirnov 1985), and can be used as an energy source for moderate plasma-surface reactions; the excitation energy $\epsilon_{\text {meta }}$ is about $6.2 \mathrm{eV}$. Figure 2 shows the electron collision cross-sections of $\mathrm{N}_{2}$ taken from Ohmori et al (1988). The target crosssection is indicated as $q_{\text {meta }}$. Its onset energy is the lowest among the electronic-state excitations of $\mathrm{N}_{2}$ under consideration, and the second lowest onset energy is $7.4 \mathrm{eV}$. Let us call the energy range $6.2-7.4 \mathrm{eV}$ 'the target energy range', in which qmeta lies alone, except for $q_{\mathrm{el}}$. The presence of such a range contributes significantly to the selective promotion of the target reaction. 


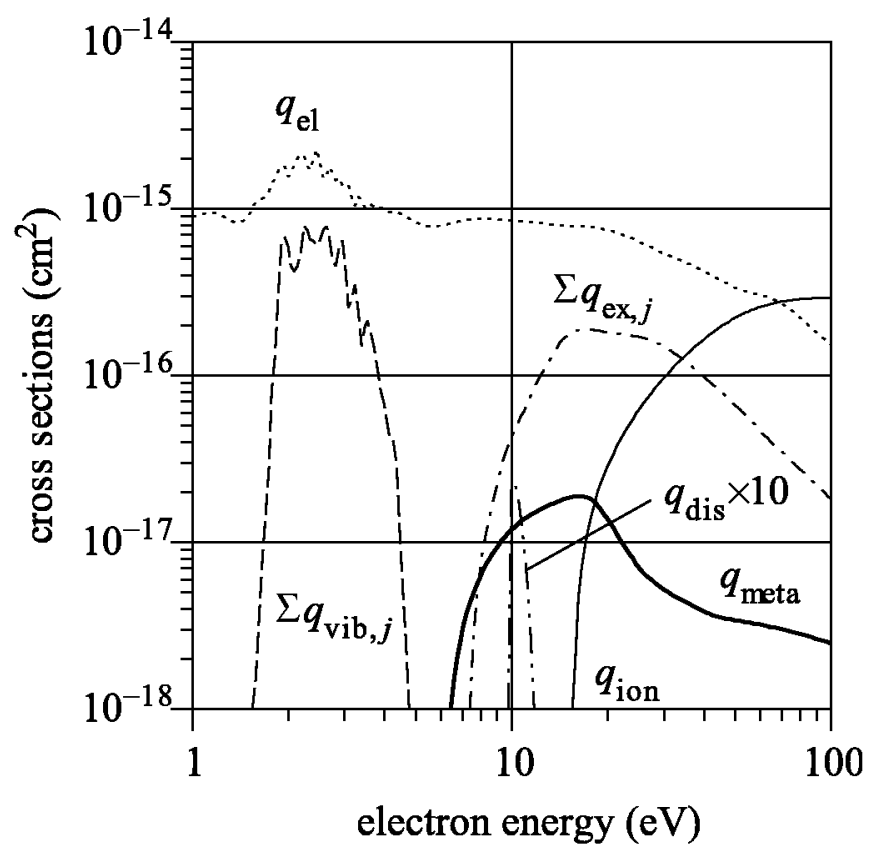

Figure 2. The electron collision cross-sections of $\mathrm{N}_{2}$ (Ohmori et al 1988). qel, elastic collision; $q_{\mathrm{vib}, j}$, vibrational excitation; $q_{\text {meta }}$, excitation to metastable state $\mathrm{A}^{3} \Sigma_{\mathrm{u}}^{+}$; $q_{\mathrm{ex}, j}$, the other electronic excitations; $q_{\mathrm{dis}}$, dissociation; and $q_{\mathrm{ion}}$, ionisation.

In order to evaluate the selectivity of the IFEA method, the energy efficiency $\eta_{\text {meta }}$ is defined as being the ratio of the electron energy utilized for the target reaction to the total energy consumption by the collisions. The efficiency is compared between the following three modes: electrons under the IFEA ( $\left.\eta_{i, \text { meta }}\right)$, electron swarms in DC drift equilibrium $\left(\eta_{e, \text { meta }}\right)$, and monoenergetic electron beams $\left(\eta_{\mathrm{b}, \mathrm{meta}}\right)$. Details of their conditions are described in following subsections.

\subsection{Impulse mode}

A Maxwellian distribution is assumed for the initial EVDF. The initial mean electron energy $\epsilon_{\text {ini }}$ is $1 \mathrm{eV}$ in the central test conditions, and IFEA at $\epsilon_{\text {ini }}=0.1 \mathrm{eV}$ is demonstrated additionally for comparison. $N$ is set to be $1.77 \times 10^{16} \mathrm{~cm}^{-3}$, which corresponds to a gas pressure of $66.5 \mathrm{~Pa}(0.5$ Torr $)$ at $0{ }^{\circ} \mathrm{C}$. The electron mean free path $\lambda \approx\left(N q_{\text {total }}\right)^{-1}$ estimated at this $N$ is about $0.06 \mathrm{~cm}$, roughly approximating $q_{\text {total }} \approx 10^{-15} \mathrm{~cm}^{2}$. The flight distance $L$ along the $x$-direction which an electron needs to obtain $\epsilon_{\text {meta }}$ is set to be shorter than $\lambda$; e.g. $L=\epsilon_{\text {meta }} /(e E)$ is about $0.031 \mathrm{~cm}$ at $E=200 \mathrm{~V} \mathrm{~cm}^{-1}$. Estimating from $P_{\text {coll }}$ in equation (5), about half of the initial electrons survive collisionless up to this $L$.

Figure 3 shows the waveforms of $E(t)$ assumed here. The rectangular models ideal impulses, and the triangular one simulates those with risetime. $T_{\text {eff }}$ for these waveforms is equivalent to their full-width at half-maximum; $T_{\text {eff }}=T$ for the former, and $T_{\text {eff }}=\frac{1}{2} T$ for the latter. The values of $E_{\max }$ and $T_{\text {eff }}$ we examine are listed in table 1 together with 
Table 1. Waveform parameters and maximum energy efficiency.

\begin{tabular}{llllll}
\hline Case & Waveform & $\begin{array}{l}E_{\max } \\
\left(\mathrm{V} \mathrm{cm}^{-1}\right)\end{array}$ & $\begin{array}{l}T_{\text {eff }} \\
(\mathrm{ns})\end{array}$ & $\begin{array}{l}\epsilon_{\text {ini }} \\
(\mathrm{eV})\end{array}$ & $\begin{array}{l}\max \left(\eta_{\mathrm{i}, \text { meta }}\right) \\
(\%)\end{array}$ \\
\hline A & Rectangle & 200 & $0.05-0.75$ & 1.0 & 20.2 \\
$\mathrm{~B}$ & Rectangle & 100 & $0.10-1.50$ & 1.0 & 18.3 \\
$\mathrm{C}$ & Triangle & 100 & $0.10-1.50$ & 1.0 & 17.7 \\
\hline $\mathrm{D}$ & Rectangle & 1000 & $0.01-0.15$ & 1.0 & 21.8 \\
\hline $\mathrm{E}$ & Rectangle & 200 & $0.05-0.75$ & 0.1 & 28.9 \\
\hline
\end{tabular}

a part of the calculation results of $\eta_{\mathrm{i}, \text { meta }}$. Cases $\mathrm{A}-\mathrm{C}$ are to observe the dependence of $\eta_{\mathrm{i}, \text { meta }}$ on the waveform. Cases $\mathrm{D}$ and $\mathrm{E}$ are examples to demonstrate further idealized IFEA with extremely short impulse and low electron temperature. The ranges of $T_{\text {eff }}$ are chosen depending on given $E_{\max }$ so that a parameter $E_{\max } T_{\text {eff }}$ (which gives the total acceleration) ranges $10-150 \mathrm{~V} \mathrm{~cm}^{-1} \mathrm{~ns}$. This range corresponds to the energy gain of 0.088-19.8 eV, which includes the target energy range of $6.2-7.4 \mathrm{eV}$.

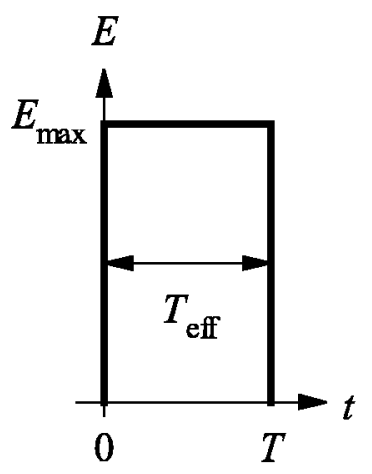

(a)

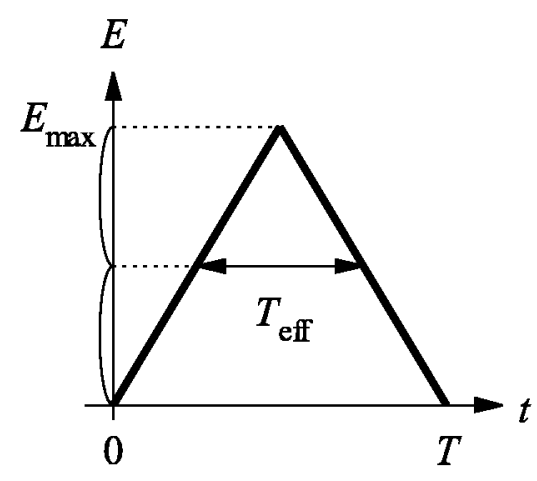

(b)

Figure 3. Impulse waveforms modelled: $(a)$, cases A, B, D and E; $(b)$, case C.

The temporal variation of the EVDF is calculated by a propagator method (Sugawara et al 1998, 2001). It is a numerical technique to solve the Boltzmann equation. The electron-molecule collisions are counted throughout the acceleration and post-acceleration periods until the electron energy becomes less than the lowest onset energy among the inelastic collisions of $\mathrm{N}_{2}$ under consideration. In this cross-section set, it is $0.29 \mathrm{eV}$ for a vibrational excitation.

The efficiency $\eta_{\mathrm{i}, \mathrm{meta}}$ is calculated as

$$
\eta_{\mathrm{i}, \mathrm{meta}}=\frac{k_{\mathrm{meta}} \epsilon_{\mathrm{meta}}}{\sum_{j} k_{j} \epsilon_{j}+\epsilon_{\mathrm{el}}^{*}} .
$$

Here, $k_{j}$ is the collision count, and $\epsilon_{j}$ is the loss energy of an inelastic collision. Subscript $j$ represents the $j$ th kind of inelastic collision, and one of the $j$ values corresponds to 
'meta' for the target reaction. $\epsilon_{\mathrm{el}}^{*}$ is the total loss energy $\epsilon_{\mathrm{el}}$ of each elastic collision. The value of $\epsilon_{\mathrm{el}}$ at an elastic collision depends on the incident electron speed $v=|\boldsymbol{v}|$ and the scattering angle. In this calculation, $\epsilon_{\mathrm{el}}$ is approximated as follows, assuming isotropic electron scattering in the cold gas model:

$$
\epsilon_{\mathrm{el}}(v)=2 \frac{m}{M}\left(\frac{1}{2} m v^{2}\right)
$$

where the factor $m / M$ represents the mass-to-mass ratio between an electron and an $\mathrm{N}_{2}$ molecule. In addition, $\epsilon_{\mathrm{el}}^{*}$ includes the electron energy remaining at the end of calculation; in other words, the residual energy is regarded as being lost by elastic collisions eventually.

\subsection{DC equilibrium mode}

The distribution function $f(v)$ of the electron speed $v=|\boldsymbol{v}|=\sqrt{v_{x}^{2}+v_{y}^{2}+v_{z}^{2}}$ in DC drift equilibrium is calculated at $E / N$ of $1-1000 \mathrm{Td}\left(1 \mathrm{Td}=10^{-17} \mathrm{~V} \mathrm{~cm} \mathrm{~cm}^{2}\right)$ by a twoterm Boltzmann equation code. $f(v)$ can be related to $f\left(v_{x}, v_{y}, v_{z}\right)$ through $f(v, \theta, \phi)$, which is the EVDF represented in a polar-coordinate form. Here, $\theta$ and $\phi$ are the polar and azimuthal angles, and $f(v), f\left(v_{x}, v_{y}, v_{z}\right)$ and $f(v, \theta, \phi)$ in drift equilibrium are independent of time. Their relation is given as follows:

$$
f(v)=\int_{\theta=0}^{\pi} \int_{\phi=0}^{2 \pi} f(v, \theta, \phi) v^{2} \sin \theta \mathrm{d} \theta \mathrm{d} \phi
$$

where $f(v, \theta, \phi)=f\left(v_{x}, v_{y}, v_{z}\right)$ at $\left(v_{x}, v_{y}, v_{z}\right)=(v \cos \theta, v \sin \theta \cos \phi, v \sin \theta \sin \phi)$.

The efficiency $\eta_{e, \text { meta }}$ is defined in the same way as $\eta_{\mathrm{i}, \text { meta }}$ in equation ( 7 ):

$$
\eta_{\mathrm{e}, \text { meta }}=\frac{\int_{0}^{\infty} \epsilon_{\mathrm{meta}} N q_{\mathrm{meta}}(v) v f(v) \mathrm{d} v}{\sum_{j} \int_{0}^{\infty} \epsilon_{j} N q_{j}(v) v f(v) \mathrm{d} v+\int_{0}^{\infty} \epsilon_{\mathrm{el}}(v) N q_{\mathrm{el}}(v) v f(v) \mathrm{d} v} .
$$

\subsection{Monoenergetic electron beam mode}

An initial energy $\epsilon_{\text {ini }}$ is assumed in common for all of the initial electrons, which are released straight towards the $+x$-direction in $\mathrm{N}_{2}$ of $N=3.54 \times 10^{16} \mathrm{~cm}^{-3}$ as a monoenergetic electron beam. The electron flight and collision are simulated by a Monte Carlo method under $E=0$ in boundary-free space. Electrons may deviate from the course of the beam after their first collisions; however, their motion and energy loss processes are traced until the electrons satisfy the same termination condition as in the impulse mode. The energy loss by elastic collision is taken into account as well as that by the inelastic collisions, and the electron scattering due to the collisions is assumed to be isotropic. We examine $\epsilon_{\text {ini }}$ of $1-20 \mathrm{eV}$, and the number of initial electrons $n_{\text {ini }}$ is 10000 at each $\epsilon_{\text {ini }}$ value. The efficiency $\eta_{\mathrm{b} \text {,meta }}$ is defined with the total initial electron energy $n_{\text {ini }} \epsilon_{\text {ini }}$ as

$$
\eta_{\mathrm{b}, \text { meta }}=\frac{k_{\text {meta }} \epsilon_{\text {meta }}}{n_{\text {ini }} \epsilon_{\text {ini }}}
$$


Here, although we have specified the $N$ value and the direction of the initial electron velocity for practical calculation, $\eta_{\mathrm{b} \text {,meta }}$ is independent of them when $E=0$ and the space simulated is boundary-free.

\section{Results and discussion}

\subsection{Electron behaviour in velocity space}

Figure 4 shows the EVDF before and after acceleration in case $\mathrm{A}$ at $T_{\text {eff }}=0.5 \mathrm{~ns}$. Here, the EVDF is depicted as the projection to the $v_{x}-v_{y}$ plane as $f\left(v_{x}, v_{y}, t\right)=$ $\int_{-\infty}^{\infty} f\left(v_{x}, v_{y}, v_{z}, t\right) \mathrm{d} v_{z} . v_{x}$ and $v_{y}$ are plotted with a scale normalized by $v_{1}$ (the electron speed associated with $1 \mathrm{eV})$, and $\left(v / v_{1}\right)^{2}$ gives the value of the electron energy in $\mathrm{eV}$. The EVDF peak decays by collisions during acceleration. The dropout electrons are scattered isotropically around $\boldsymbol{v}=0$, and form the distribution tail. They restart acceleration following the leading peak for the residual impulse period, but they are out of control by the IFEA method. Electrons forming the leading peak continue the collective motion in velocity space, and those without collisions reach the target energy range.

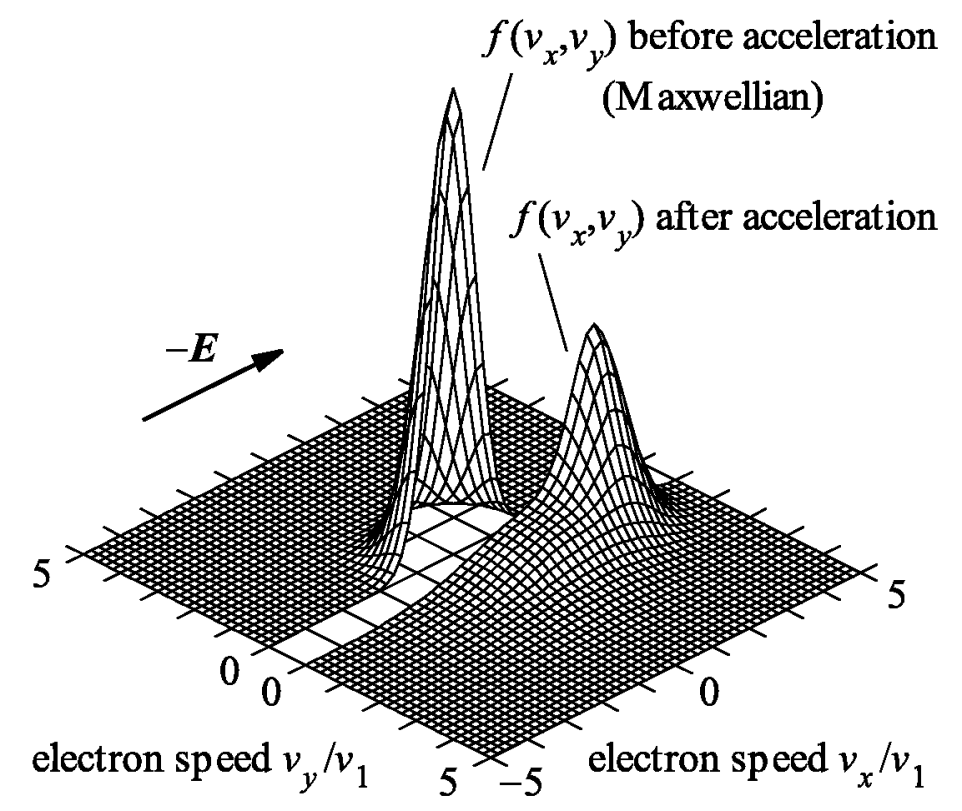

Figure 4. The EVDF $f\left(v_{x}, v_{y}, t\right)$ before and after acceleration (case A, $T_{\text {eff }}=0.5 \mathrm{~ns}$ ). Each is symmetric in $v_{y}=0$, then is depicted only for $v_{y} \geq 0$. The scale unit $v_{1}$ is the electron speed associated with $1 \mathrm{eV}$.

Figures 5 and 6 show the cross-sections of the EVDF at $v_{y}=0$ in cases $\mathrm{A}$ and D. $E_{\max } T_{\text {eff }}=100 \mathrm{~V} \mathrm{~cm}^{-1} \mathrm{~ns}$ in both cases $\left(T_{\text {eff }}=0.5 \mathrm{~ns}\right.$ in case A, and $0.1 \mathrm{~ns}$ in case D). The first six curves in each figure are those during acceleration, and the others are those after acceleration. The peak in case $\mathrm{D}$ decays less than in case A because of 
fewer collision opportunities due to the shorter acceleration period. The decay is not simply exponential because $q_{\text {total }}$ is energy dependent. Especially in figure 5, a large decay appears between $t=0.2$ and $0.3 \mathrm{~ns}$. This is due to scattering by the $q_{\mathrm{el}}$ and $q_{\mathrm{vib}}$ peaks lying around $\epsilon=2 \mathrm{eV}\left(v / v_{1}=1.4\right)$. This influence particular to $\mathrm{N}_{2}$ is called the barrier effect (Engelhardt et al 1964, Sugawara et al 2001), which functions to prevent slow electrons from accelerating. After the impulse field is turned off, the peak moves back towards $v_{x}=0$ for energy loss by collision. The electrons concentrate around $v=0$ again after a cooling period over $10 \mu \mathrm{s}$.

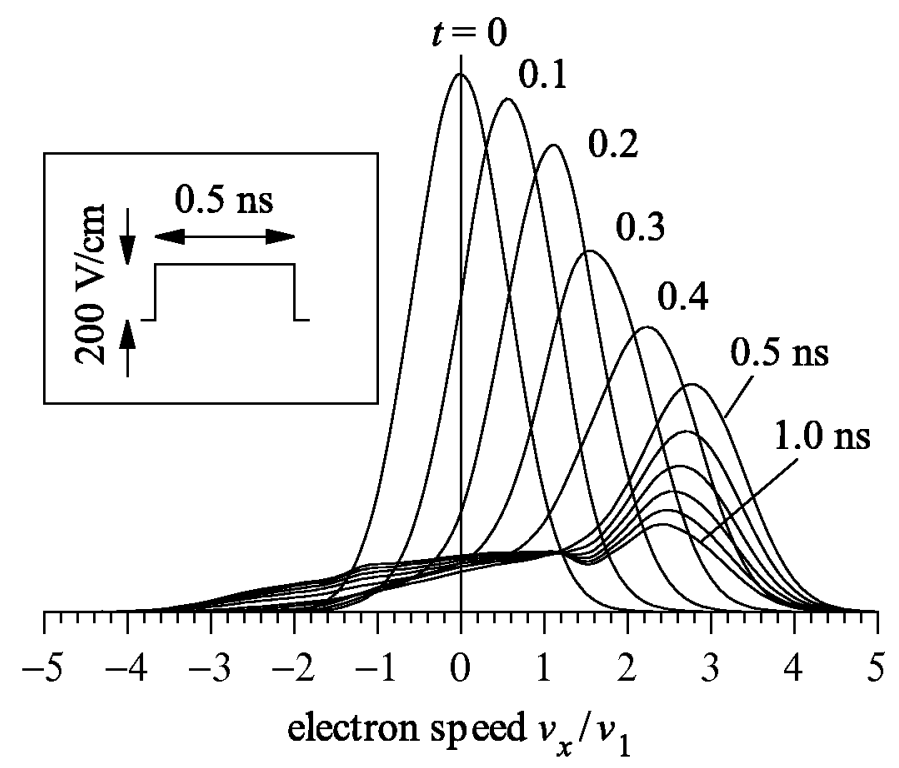

Figure 5. The EVDF $\left.f\left(v_{x}, v_{y}, t\right)\right|_{v_{y}=0}$ at $t=0-1.0$ ns (0.1 ns step); case A, $T_{\mathrm{eff}}=0.5 \mathrm{~ns}$.

Figure 7 shows loci of the EVDF peak in cases A-D at $E_{\text {max }} T_{\text {eff }}=100 \mathrm{~V} \mathrm{~cm}^{-1} \mathrm{~ns}$. The relative peak height represents the ratio of the electrons remaining free from collisions. The decay becomes larger as $T$ increases. Compared with cases $\mathrm{A}-\mathrm{C}$, case $\mathrm{D}$ at a shorter $T$ is much less influenced by collisions. Electrons can evade the barrier effect of the $q_{\mathrm{el}}$ and $q_{\mathrm{vib}}$ peaks when the impulse is short and intense.

\subsection{Comparison of energy efficiencies in different modes}

Figures 8-10 show $\eta_{\mathrm{i}, \text { meta }}, \eta_{\mathrm{e}, \text { meta }}$ and $\eta_{\mathrm{b} \text {,meta }}$ together with the energy consumption ratios of other collision processes. In figure $8, \eta_{\mathrm{i} \text {,meta }}$ reaches $20 \%$. This value is higher than the maximum value of $\eta_{\mathrm{e} \text {,meta }}$ of about $10 \%$ in figure 9 . The selectivity in the equilibrium mode is low for dispersed EVDF because different kinds of inelastic collisions occur simultaneously in a wide energy range. In contrast, electrons in the impulse mode are more concentrated around the target energy range, which leads to higher energy efficiencies. This is indicated by the result that the maximum values of $\eta_{\mathrm{i}, \mathrm{meta}}$ appear around a specific value of $E_{\max } T_{\text {eff }}=84 \mathrm{~V} \mathrm{~cm}^{-1}$ ns which corresponds to the energy gain 


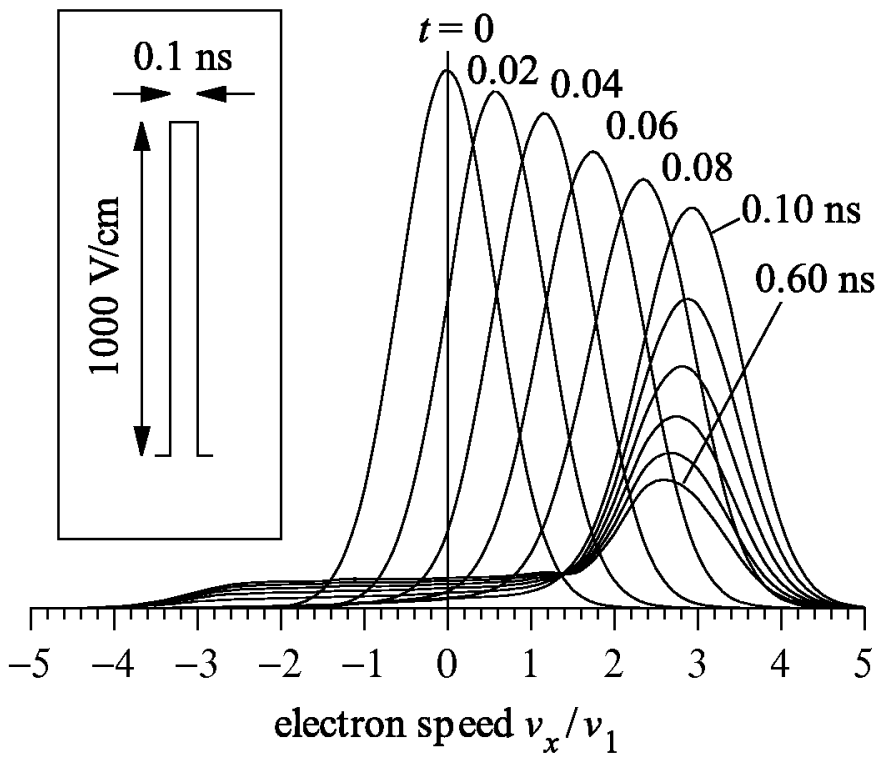

Figure 6. The EVDF $\left.f\left(v_{x}, v_{y}, t\right)\right|_{v_{y}=0}$ at $t=0-0.1 \mathrm{~ns}(0.02 \mathrm{~ns}$ step) and $t=0.1-0.6 \mathrm{~ns}$ (0.1 ns step); case D, $T_{\mathrm{eff}}=0.1 \mathrm{~ns}$.

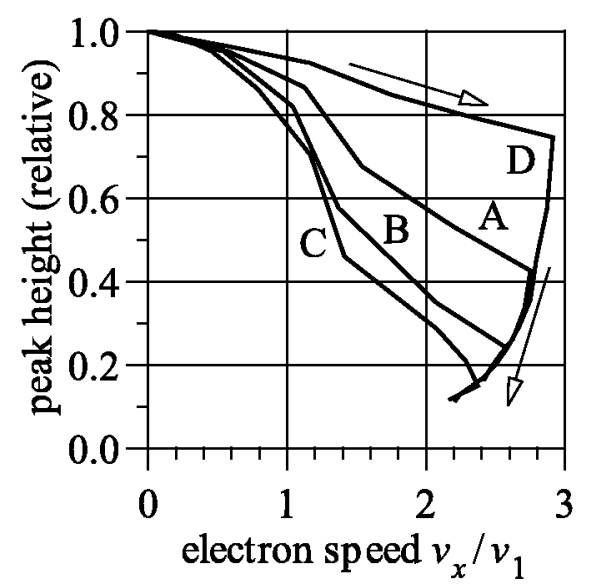

Figure 7. Comparison of loci of the EVDF peaks in cases $\mathrm{A}-\mathrm{D}$ at $E_{\max } T_{\mathrm{eff}}=$ $100 \mathrm{~V} \mathrm{~cm}^{-1} \mathrm{~ns}$.

of $\epsilon_{\text {meta }}=6.2 \mathrm{eV}$. At higher $E_{\max } T_{\text {eff }}$ values, $\eta_{\mathrm{i}, \text { meta }}$ decreases because of over-acceleration that induces inelastic processes other than the target reaction.

The merit of concentrated EVDF emerges dramatically in the beam mode. In figure $10, \eta_{\mathrm{b}, \text { meta }}$ is over $80 \%$ when $\epsilon_{\mathrm{ini}}$ is in the target range $6.2-7.4 \mathrm{eV}$. Although elastic collision is more than 100 times as frequent as the target process in this energy range, the energy loss by elastic collision is not significant because of a small $m / M$ of only about $2 \times 10^{-5}$. The electrons hold most of their initial energy until the target reaction occurs even after hundreds of elastic collisions. This result suggests improvement of $\eta_{\mathrm{i} \text {,meta }}$ by making the EVDF in the impulse mode more monoenergetic. A possible way 


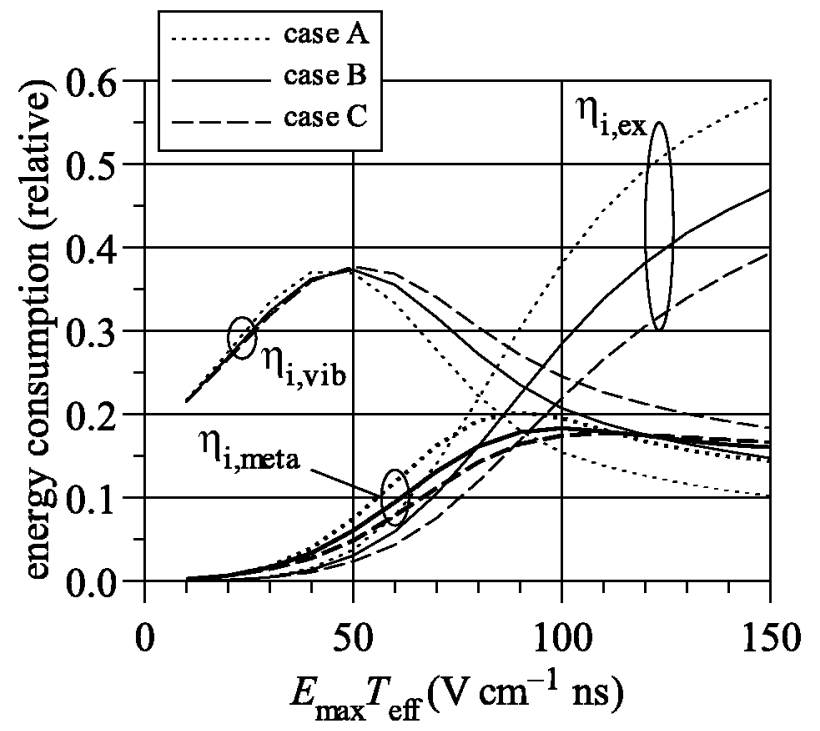

Figure 8. Energy consumption ratios in the impulse field mode in cases A, B and C (see table 1 for detailed conditions).

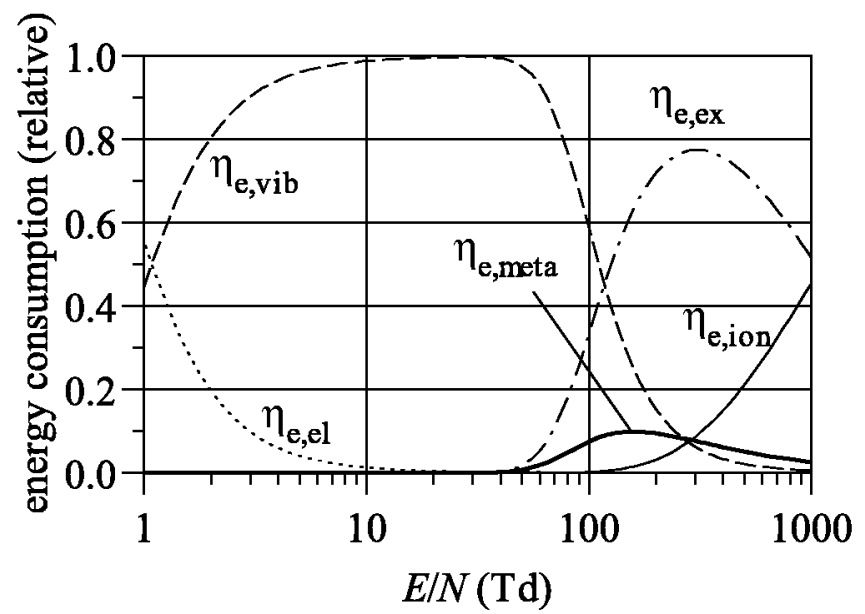

Figure 9. Energy consumption ratios in the DC drift equilibrium mode.

would be to reduce the scattering during the IFEA with shorter and intenser impulse. Another would be to make the electron temperature before the IFEA lower. Results of such attempts are presented in the next subsection.

\subsection{Dependence of energy efficiency on impulse waveform and electron temperature}

In figure 8, curves of $\eta_{\mathrm{i}, \text { meta }}$ for cases $\mathrm{A}-\mathrm{C}$ show that $\eta_{\mathrm{i}, \text { meta }}$ increases as $T$ becomes shorter in a parameter range of $E_{\max } T_{\text {eff }}<100 \mathrm{~V} \mathrm{~cm}^{-1} \mathrm{~ns}$. This tendency is understood as decrease of the electron dropout, as explained before. Because the dropout leaves electrons unaccelerated, this understanding is supported also by the decrease in $\eta_{\mathrm{i}, \mathrm{vib}}$; vibrational excitation occurs mainly at low energies. Keeping this tendency, a higher 


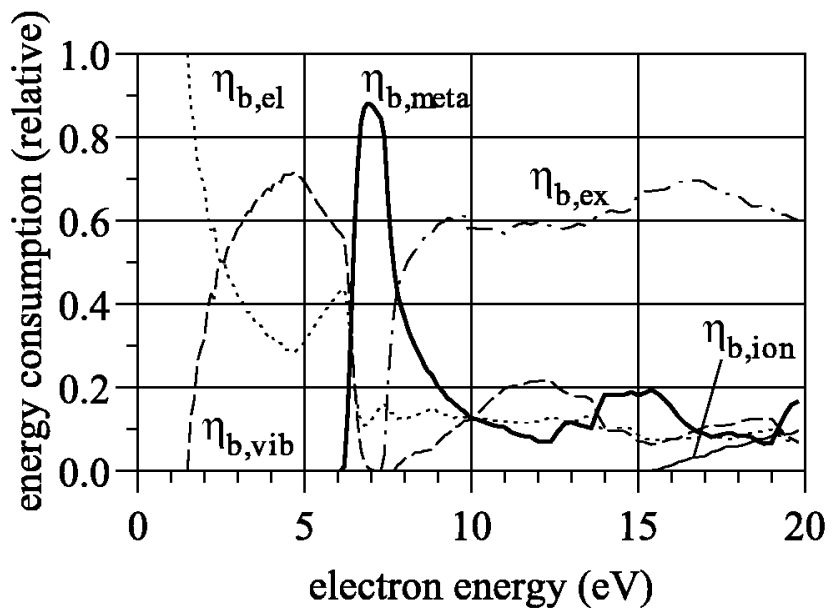

Figure 10. Energy consumption ratios in the monoenergetic electron beam mode. The horizontal axis represents the initial energy of electrons in the beam.

$\eta_{\mathrm{i}, \text { meta }}$ of $22 \%$ is obtained in case D in figure 11 under an extremely short impulse.

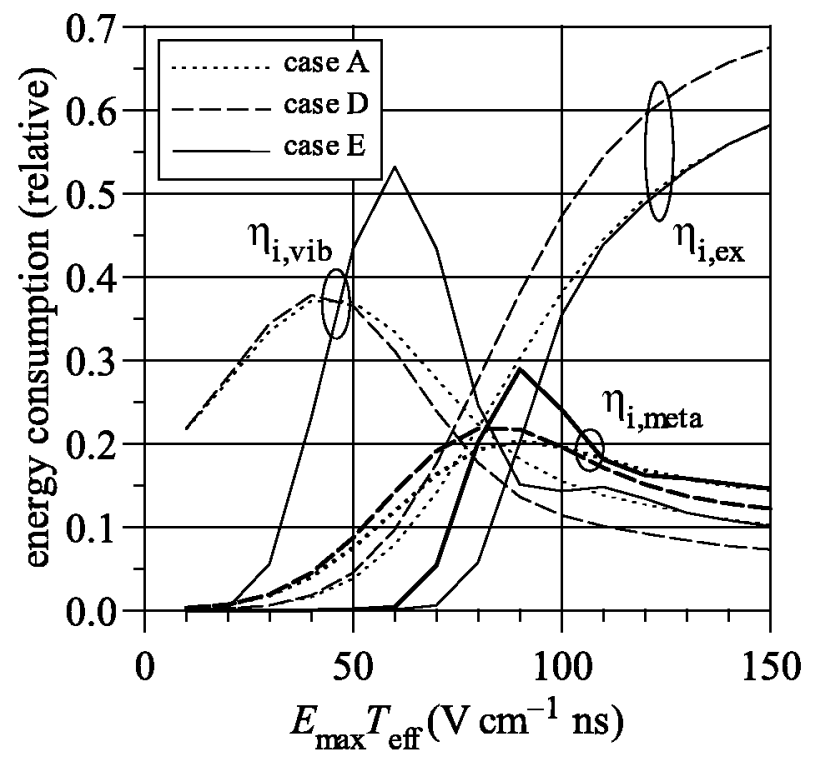

Figure 11. Energy consumption ratios in the impulse field mode in cases A, D and $\mathrm{E}$ (see table 1 for detailed conditions).

However, it is at a lower electron temperature that a significant improvement of the energy efficiency is achieved. In case $\mathrm{E}$ at $\epsilon_{\mathrm{ini}}=0.1 \mathrm{eV}, \eta_{\mathrm{i} \text {,meta }}$ reaches $29 \%$ (figure 11). As indicated by a sharp rise of $\eta_{i, \text { meta }}$, the selectivity in the impulse mode is high and sensitively dependent on $E_{\max } T_{\text {eff }}$ at low electron temperature.

When the IFEA is driven in repetitive pulse mode, some efficient electron cooling mechanism would be necessary to keep a high selectivity by recovering a low electron temperature before applying succeeding impulses. For this purpose, admixture of 
coolant species to the gas could be supposed. However, because the IFEA method is a technique to avoid the EVDF dispersion and has no active function to make the EVDF concentrated, we leave the cooling issue for future investigation elsewhere.

\subsection{Possibility of large volume reaction by the IFEA method}

As a potential application of the IFEA method, large volume reaction in a plasma bulk is considered here. Electrons in plasma bulks are usually at a low temperature and a high concentration, which is suitable for the IFEA method, and mass production of $\mathrm{N}_{2}\left(\mathrm{~A}^{3} \Sigma_{\mathbf{u}}^{+}\right)$is expected. In order to perform IFEA in plasma bulk, we need satisfactory penetration of the impulse electric field into the bulk. The impulse risetime must be shorter than the plasma response time, otherwise the impulse field would be shielded by the plasma sheath. The period of plasma oscillation (i.e. the inverse of the plasma frequency $f_{\mathrm{p}}$ ) can be a measure to evaluate the plasma response time:

$$
\frac{1}{f_{\mathrm{p}}}=2 \pi \sqrt{\frac{\epsilon_{0} m}{e^{2} n_{\mathrm{e}}}} .
$$

Here, $\epsilon_{0}$ is the permittivity of free space, and $n_{\mathrm{e}}$ is the electron concentration. At a typical value of $n_{\mathrm{e}} \sim 10^{9} \mathrm{~cm}^{-3}$ for $\mathrm{N}_{2}$ radio frequency $(13.56 \mathrm{MHz})$ plasmas, we obtain $1 / f_{\mathrm{p}}=3.5 \mathrm{~ns}$. The impulses investigated in this paper are shorter than this value, which suggests a fair prospect of the IFEA in plasma bulks.

\section{Conclusion}

We have presented a theoretical basis of the IFEA in gas, and have demonstrated selective production of $\mathrm{N}_{2}\left(\mathrm{~A}^{3} \Sigma_{\mathbf{u}}^{+}\right)$by electron-impact excitation of $\mathrm{N}_{2}$ as an example of the IFEA application.

Under a short intense electric field, electrons in gas can be accelerated in a practically collisionless condition, and the concentration of the electrons in the velocity distribution is kept during the IFEA. Controlling the impulse field strength and duration, one can feed a bunch of electrons to an energy range in which this target process (i.e. excitation of $\mathrm{N}_{2}\left(A^{3} \Sigma_{\mathrm{u}}^{+}\right)$) occurs efficiently. The electron energy efficiency has been defined to quantify the reaction selectivity, and it has been shown that the efficiency of the IFEA method reaches to $20-30 \%$. These values are higher than that in electron swarms in DC drift equilibrium (i.e. less than 10\%).

The high selectivity of the IFEA method is based on the concentration of the electron velocity distribution. Therefore, the selectivity is high and its dependence on the impulse property is sharp when the electron temperature before IFEA is low.

\section{Acknowledgments}

The authors wishes to thank Professor S Katsuki of Kumamoto University for providing references and comments on ultra-short high voltage pulses. 


\section{References}

Anikin N B, Pancheshnyi S V, Starikovskaia S M and Starikovskii A Yu 1998 J. Phys. D: Appl. Phys. $31826-33$

Carman R J and Mildren R P 2003 J. Phys. D: Appl. Phys. 36 19-33

Engelhardt A G, Phelps A V and Risk C G 1964 Phys. Rev. 135 A1566-74

Katsuki S, Iyonaga N, Takano D, Namihira T and Akiyama H 2001 Proc. 28. IEEE Int. Conf. Plasma Sci. and 13. IEEE Int. Pulsed Power Conf. 2 1102-5

Macheret S O, Shneider M N and Miles R B 2002 IEEE Trans. Plasma Sci. 30 1301-14

Ohmori Y, Shimozuma M and Tagashira H 1988 J. Phys. D: Appl. Phys. 21 724-9

Pancheshnyi S V, Starikovskaia S M and Starikovskii A Yu 1999 J. Phys. D: Appl. Phys. 32 2219-27

Penetrante B M, Hsiao M C, Bardsley J N, Merritt B T, Vogtlin G E, Kuthi A, Burkhart C P and Bayless J R 1997 Plasma Sources Sci. Technol. 6 251-9

Radzig A A and Smirnov B M 1985 Reference Data on Atoms, Molecules, and Ions (Berlin: SpringerVerlag) 386

Starikovskaia S M and Starikovskii A Yu 2001 J. Phys. D: Appl. Phys. 34 3391-9

Sugawara H, Sakai Y, Tagashira H and Kitamori K 1998 J. Phys. D: Appl. Phys. 31 319-27

Sugawara H, Satoh K and Sakai Y 2001 J. Phys. D: Appl. Phys. 34 3191-6

Sugawara H, Shimoda T and Sakai Y 1999 Abst. Int. Symp. on Electron-Molecule Collisions and Swarms: A Satellite of 21st Int. Conf. on Physics of Electronic and Atomic Collisions (Tokyo, Japan, 18-20 July) 49-50

Sugawara H, Tagashira H and Sakai Y 1997 J. Phys. D: Appl. Phys. 30 368-73

Vollkommer F and Hitzschke L 1997 US patent 5604410

Vollkommer F and Hitzschke L 1998 Proc. 8th Int. Symp. on Science and Technology of Light Sources (LS-8) (Greifswald, Germany, 30 August-3 September) IL-07 pp 51-60 